\title{
ABRE LOS OJOS: ¿ACTUALIZACIÓN O REESCRITURA DE LA VIDA ES SUEÑO?
}

\author{
CARMen Rivero \\ Westfälische Wilhelms- Universität Münster
}

Title: Updating or Rewriting Life Is a Dream?: Open Your Eyes by Alejandro Amenábar

\begin{abstract}
The present study analyzes the relationship between Life is a dream by the Baroque playwright Calderón de la Barca and Alejandro Amenábars Abre los ojos from an intermedial perspective. By way of focusing not only on the similarities but also on the divergences in the treatment of the topos of life as a dream, it concludes that Amenábar's film represents a revision of the Calderonian conception of existence. Amenábar presents an almost inverted perspective on the central axes of reflection that underpin Life is a dream: the relationship between dream and reality and the question of human freedom.
\end{abstract}

Key words: Intermediality. Calderón. Life is a dream. Amenábar. Open your eyes.

Con el segundo de sus largometrajes, Abre los ojos, Alejandro Amenábar consolida uno de los rasgos definitorios de su trayectoria cinematográfica, a saber, la integración de cine comercial y de autor; de la tradición hollywoodiense y la europeo-española; ${ }^{1}$ de teatro y cine. ${ }^{2}$ El presente estudio centra su atención en estos últimos dos aspectos para analizar la posible deuda del cineasta español con el drama filosófico La vida es sueño del dramaturgo barroco español, Calderón de la Barca. Han sido similitudes temáticas, ${ }^{3}$ sobre todo en relación a la intersección de planos entre sueño

${ }^{I} R$. Maule, "Cultural Specificity and Transnational Address in the New Generation of Spanish Film Authors: The Case of Alejandro Amenábar", C. Sánchez-Conejero (ed.), Spanishness in the Spanish Novel and Cinema of the 2oth -2 ist Century, Newcastle: Cambridge Scholars Publishing, 2007, pp. 107-I 20, aquí: p. I I 5.

${ }_{2}^{2}$ A. Amenábar, Abre los ojos. Madrid: Sociedad General de Cine, I997, Comentarios del director.

${ }^{3}$ S. Robertson, "Life is virtual dream: Amenábar Reading Calderón", G. CabelloCastellet, J. Martí-Olivella y G. H. Wood (eds.), Cine-Lit 20oo. Essays on Hispanic Film and Fiction, Corvallis: Oregon State University, 2000, pp. I I 5-I25, aquí: p. I I 8. 
y realidad, ${ }^{4}$ las que han llevado a la crítica a establecer una filiación entre ambas obras.

Calderón presentaba en escena la historia de Segismundo, un príncipe al que su padre, el rey Basilio, había encerrado en una torre, para evitar el cumplimiento de una profecía que aseguraba que su hijo se convertiría en tirano y traería consigo la desgracia del reino. El joven príncipe logra, sin embargo, sobreponerse al destino que las estrellas habían previsto para él y devenir, contra todo pronóstico, un buen rey a través de sus virtuosas acciones. Calderón, según Alfonso Reyes, se valía, de este modo, “de un bello recurso dramático para conceder dos vidas distintas a Segismundo [la de príncipe y la de prisionero] aunque ligadas ambas por el recuerdo", una estructura sobre la que Amenábar construye también la historia de su protagonista.

Al igual que Segismundo ha nacido príncipe, también César, el protagonista de Amenábar, es, según Robertson, una especie de príncipe moderno, rico por herencia, un privilegiado frente a la mayoría, cuyo palacio es un lujoso apartamento de pulidos suelos y hermosos espejos". ${ }^{6}$ Como en la obra calderoniana, ambos pierden la libertad a causa de la acción tiránica de un tercero: en Calderón, el padre de Segismundo, que encierra a su hijo en una torre para evitar el cumplimiento de la profecía; en la película de Amenábar, Nuria, una amante despechada que, a través de un accidente de coche intencionado, provocará el encerramiento de César tanto en la vida

${ }^{4}$ J. Xuan, "Calderón y el cine: La vida es sueño y Abre los ojos de Alejandro Amenábar", M. Tietz (ed.), Teatro calderoniano sobre el tablado. Calderón y su puesta en escena a través de los siglos, Stuttgart: Franz Steiner Verlag, 2003, pp. pp. 479-491, aquí: p. 480; D. Perri, "Amenábars Abre los ojos: The post-human subject". Hispanófila, I 54 (2008), pp. 89-98, aquí: p. 9 I.

${ }^{5}$ A. Reyes, "Un tema de La vida es sueño", A. Reyes, Obras completas, VI (pp. I 82-248), México: Fondo de Cultura Económica, I957, p. 238.

${ }^{6}$ S. Robertson, "Life is virtual dream: Amenábar Reading Calderón", G. CabelloCastellet, J. Martí-Olivella y G. H. Wood (eds.), Cine-Lit 20oo. Essays on Hispanic Film and Fiction (pp. I I 5-I 25), Corvallis: Oregon State University, 2000, pp. I I 5-I 25, aquí: I I 8. 
real como en la soñada. En la vida real, el protagonista quedará atrapado en un cuerpo deformado que, tras el accidente, ya no reconoce como suyo ${ }^{7}$ mientras en el sueño el traumático recuerdo, reprimido en el subconsciente, lo mantendrá encerrado en un psiquiátrico penitenciario.

A causa del fatal choque, la cara de César ha quedado totalmente deformada, mezclándose, a partir de ese momento, escenas de una vida real, en la que debe vivir con la pérdida de su estatus físico y social tras el accidente, y de una onírica, en la que sueña que la vida continúa como si el accidente no hubiera tenido lugar o sus consecuencias hubieran sido remediadas. Esta vida onírica le es proporcionada por los servicios de una compañía llamada Life Extension, que se ofrece, bien a congelar su cuerpo hasta un futuro en el que su deformación facial pueda ser operada, bien a proporcionarle una vida virtual idealizada, en la que todo recuerdo traumático ha sido eliminado de su memoria. Como en el caso de Segismundo, aunque desde una perspectiva más futurista, también la vida de César se representa, en principio, a partir de la interacción entre sueño y realidad.

A pesar de la recuperación de la temática de la vida como sueño, que invita a la legítima vinculación realizada por la crítica entre la obra calderoniana y la película de Amenábar, el planteamiento del cineasta difiere sustancialmente, con todo, tal y como se mostrará a continuación, del que lleva a escena el dramaturgo áureo.

Estudios como, entre otros, el de Robertson, parten de la idea, por el contrario, de que la película retoma y reconfigura "los mismos dilemas morales que Calderón de la Barca presentaba a sus espectadores del siglo XVII ”. ${ }^{8}$ Pero ¿cómo podría tener un espectador del siglo XX los mismos

7Una idea que Amenábar desarrollará posteriormente, en relación a la eutanasia, en Mar adentro.

${ }^{8}$ S. Robertson, "Life is virtual dream: Amenábar Reading Calderón”, G. CabelloCastellet, J. Martí-Olivella y G. H. Wood (eds.), Cine-Lit 2ooo. Essays on Hispanic Film and Fiction (pp. I I 5-I 25), Corvallis: Oregon State University, 2000, pp. I I 5-I 25, aquí: p. I I 5 . 
dilemas morales que en el siglo XVII? Tratarán de exponerse, por tanto, en lo que sigue, las diferencias fundamentales entre la obra calderoniana y la película de Amenábar para llegar, finalmente a la conclusión de que la película no es una actualización sino, más bien, una revisión de los ejes fundamentales de la obra calderoniana.

Mientras en Calderón, la interacción de sueño y realidad se presenta a modo de alegoría de la cosmovisión barroca como coincidentia oppositorum, ${ }^{9}$ en Amenábar el sueño ilustra, más bien, el funcionamiento del aparato psíquico freudiano. El sueño cumple la función, entonces, de liberar los recuerdos traumáticos reprimidos en el subconsciente y posee, desde esta perspectiva, una función curativa y liberadora. ${ }^{\circ}$ En todo sueño, se reclama al "yo" la satisfacción de un deseo insatisfecho, que el "yo" durmiente trata de cumplir simulando su concesión. ${ }^{\text {I }}$ Mientras el sueño, entonces, posee en Calderón una dimensión fundamentalmente existencial y alegórica, en Amenábar este ilustra, más bien, el funcionamiento de la psique humana.

Por otra parte, y como ha sido repetido en numerosas ocasiones, en la obra calderoniana, realidad y sueño no dejan nunca de conformar nunca una unidad. ${ }^{\mathrm{I}}$ Amenábar, en cambio, subraya su estricta separación en la escena final de la película, en la que César elige la realidad, abandonando, con ello, el sueño. Este, construido a partir del olvido de una experiencia traumática del pasado borrada de la memoria como si de un dispositivo elec-

${ }^{9}$ D. Alonso, Poesía española. Ensayo de métodos y límites estilísticos, Madrid: Gredos, I 976, pp. 388-389.

IoS. Freud, Die Traumdeutung, Köln: Anaconda, 20 I4, p. 7 I.

${ }^{\text {II }}$ S. Freud, Abriss der Psychoanalyse, Stuttgart: Reclam, 20 io, p. 37.

${ }^{12}$ Sciacca constituye, en este sentido, una excepción cuando objeta que los que, como Segismundo, tienen conciencia de que vivir en el mundo es soñar no sueñan (M. F. Sciacca, "Verdad y sueño de La vida es sueño de Calderón de la Barca", M. Durán (ed.), Calderón y la crítica. Historia y antología, I (pp. 54I-562). Madrid: Gredos, I 976, pp. 54I-562, aquí: p. 544). Calderón, sin embargo, desarrolla una idea similar en El gran teatro del mundo. El hombre que sabe que la vida es teatro no deja de ser un actor sino que sabe que, como tal, ha de desempeñar adecuadamente su papel. De modo paralelo, Segismundo aprende que, aunque la vida sea sueño, obrar bien es lo que importa para ganarse la salvación. 
trónico de memorización se tratara, estaba desde un comienzo condenado al fracaso, porque, de acuerdo a Freud: "Nada de lo que hayamos poseído espiritualmente se pierde nunca del todo [... $\mathrm{O}$ O como afirma Delbœuf: 'que toda impresión, incluso la más insignificante, deja una huella inalterable, indefinidamente susceptible de reaparecer"'. ${ }^{13}$ La técnica no podrá burlar, a pesar de sus vanas promesas, el funcionamiento natural del sueño que, inevitablemente, intentará acercarlo una y otra vez al recuerdo reprimido: ${ }^{14}$ Nuria y el accidente.

La neurosis de César surge a partir del contenido del ello que busca liberarse mientras el yo se resiste. El sueño acaba constituyendo el medio, entonces, para su liberación a través de una regresión del aparato psíquico. ${ }^{\text {Is }}$ César necesita, para ello, de alguien que establezca la relación entre el contenido manifiesto y el latente de su sueño. Esta es, precisamente, la función que asumen tanto su psiquiatra, Antonio, como el director de la compañía Life Extension, que interviene en el sueño del protagonista para explicarle su significado y permitirle, finalmente, recordar. Al final del proceso se produce una escisión, entre una parte de la psique, que acepta la realidad, y otra, en la que la influencia de los impulsos la aleja de ella. ${ }^{16}$ Desde esta perspectiva, el protagonista es situado en la secuencia de la azotea entre la realidad y el sueño. Al no estar sujeto este último, sin embargo, a su control, el protagonista decide abrir los ojos; despertar.

La distinta concepción del sueño, en el primer caso alegórico, en el segundo, psíquico, ha llevado a ambas obras, en definitiva, a desenlaces

I3"Nichts, was wir geistig einmal besessen, ganz und gar verloren werden kann [...]. Oder, wie Delboeuf es ausdrückt, "que toute impression même la plus insignifiante, laisse une trace inaltérable, indéfiniment susceptible de reparaitre au jour”" (S. Freud, Die Traumdeutung, Köln: Anaconda, 2014, p. 25). La traducción en el texto es mía.

${ }^{14}$ S. Freud, Die Traumdeutung, Köln: Anaconda, 2014, pp. 55, 377.

${ }^{15}$ S. Freud, Abriss der Psychoanalyse, Stuttgart: Reclam, 20 Io, p. 32; S. Freud, Die Traumdeutung, Köln: Anaconda, 2014, p. 35 I.

${ }^{16}$ S. Freud, Abriss der Psychoanalyse, Stuttgart: Reclam, 20 Io, p. 77. 
opuestos. La lección aprendida por Segismundo es, como indica el propio título de la obra, que la vida es sueño. Calderón muestra cómo la vida terrena, al contrario que la ultraterrena, es apariencia y engaño. Su buen príncipe cristiano sabe, en consecuencia, que la primera no es sino vanitas y que no despertará de ese sueño hasta alcanzar la segunda. El objetivo de la película de Amenábar no es, sin embargo, aprender como lo hace el príncipe Segismundo, que la vida es sueño o apariencia, tal y como ha afirmado Perriam. ${ }^{17}$ En Abre los ojos, el mensaje contrario queda claro desde el mismo título (Abre los ojos). Se trata, ahora de mostrar que la vida no es sueño y, si la huida de una experiencia traumática la convierte en ello, conviene despertarse cuanto antes, porque los mecanismos del subconsciente lo convertirán indefectiblemente en pesadilla.

La distinta concepción del sueño implica, asimismo, su distinto funcionamiento en ambas obras. Mientras el sueño alegórico de Segismundo está dirigido por una razón que somete a los instintos, el de César, por el contrario, que está realmente dormido, está dirigido por los impulsos del inconsciente.

La razón por sí sola no basta, con todo, a Segismundo, porque el hombre, para perfeccionarse, necesita la ayuda de otros hombres; necesita vivir en sociedad. En ello se basan Rico e Ynduráin para establecer en La vida es sueño la influencia de De regimine principum, de Santo Tomás de Aquino, ${ }^{18}$ mientras Strosetzki recuerda cómo la idea procede, originariamente, de Aristóteles: frente a los conocimientos teóricos, que pueden adquirirse a través del estudio, la virtud moral no puede obtenerse sin la

${ }^{17}$ Ch. Perriam, "Alejandro Amenábar's Abre los ojos/Open your eyes (1 997)”, A. LázaroReboll y A. Willis (eds.), Spanish popular cinema, Manchester: Manchester University Press, 2004, pp. 209-22 I, aquí: pp. 2 I 2-2 I 3.

${ }^{18 D}$. Ynduráin, D.; F. Rico, F., "El monólogo de Segismundo”, F. Rico (coord.), Historia y crítica de la literatura española, 3/ I, Barcelona: Crítica, I 992, pp. 436-443, aquí: p. 437. 
práctica. ${ }^{19}$ Por ello, aunque Clotaldo trata de instruir a Segismundo en las ciencias y en la ley cristiana durante su encerramiento en la torre, ${ }^{20}$ Segismundo, privado de la comunicación con otros hombres, solo puede comportarse de manera violenta. Segismundo muestra, así, tal y como afirma Picatoste, la ineficacia del estudio fuera de la vida en sociedad. ${ }^{21}$

Todo ello justifica que las primeras reacciones de Segismundo en su primera salida de la torre sean puramente animales. Tira a un criado por la ventana y amenaza con hacer lo mismo con el honor de Rosaura. ${ }^{22}$ Sin embargo, Segismundo es hombre y no fiera y posee, por tanto, una razón que acaba sometiendo los instintos a su control. Así, se reporta primero ante el instinto de matar a Clotaldo:

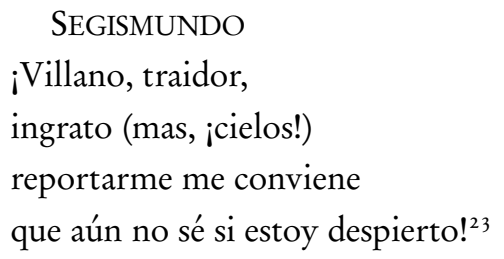

Ya en la escena final queda claro que Segismundo, definitivamente, ha aprendido la lección. En lugar de dejarse llevar por la ira y vengarse de su padre, reflexiona, comprende su error y decide, de acuerdo al cuarto mandamiento, honrarle con humildad. La noble acción del hijo provoca admiración en el padre:

${ }^{19}$ Ch. Strosetzki, "El Segismundo de Calderón y la segunda naturaleza”, Anuario Calderoniano, 4 (20I I), pp. 333-347.

${ }^{20} \mathrm{P}$. Calderón de la Barca, La vida es sueño, Ed. Fausta Antonucci, Barcelona: Crítica, 2008, p. I 43.

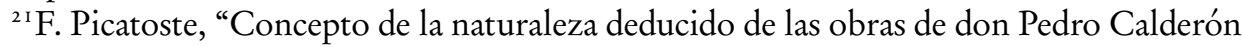
de la Barca”, M. Durán (ed.), Calderón y la crítica. Historia y antología, I, Madrid: Gredos, i 976, pp. i66-248, aquí: p. 247.

${ }^{22}$ P. Calderón de la Barca, La vida es sueño, Ed. Fausta Antonucci, Barcelona: Crítica, 2008, p. I79.

${ }^{23}$ P. Calderón de la Barca, La vida es sueño, Ed. Fausta Antonucci, Barcelona: Crítica, 2008, p. 2 II. 
BASILIO

$[\ldots]$ príncipe eres.

A ti el laurel y la palma

se te deben. Tú venciste:

corónente tus hazañas. ${ }^{24}$

La pieza muestra cómo Segismundo se ha servido de la experiencia vivida para no cometer los mismos errores una vez que ha alcanzado el po$\operatorname{der}^{25} \mathrm{y}$, así, cuando llega el momento, sabe renunciar a lo que los instintos biológicos le impulsan a hacer. ${ }^{26}$ Es a través del uso de la razón, que Segismundo domina, finalmente, sus pasiones y, se convierte, de este modo, en un buen príncipe cristiano ${ }^{27}$ que vela por el bien común tras haber renunciado al particular.

En la película de Amenábar, sin embargo, el sueño es dirigido por el inconsciente, que opera, para desesperación del protagonista, conforme a sus propias reglas y al margen de la lógica. Por este motivo, el contenido manifiesto del sueño es con frecuencia absurdo. ${ }^{28}$ Buen ejemplo de ello es que los contrarios dejan de encontrarse en relación de oposición. En el contenido manifiesto del sueño estos pueden llegar incluso a identificarse, de manera que cada elemento puede ser potencialmente representado por su contrario. ${ }^{29}$ De acuerdo a las tendencias características de condensación y traslación del sueño, la amada Sofía es sustituida por la odiada Nuria, causante de su desgracia.

\footnotetext{
${ }^{24}$ P. Calderón de la Barca, La vida es sueño, Ed. Fausta Antonucci, Barcelona: Crítica, 2008, p. 245.

${ }^{25}$ F. Antonucci, "Introducción”, P. Calderón de la Barca, La vida es sueño, Ed. Fausta Antonucci, Barcelona: Crítica, 2008, p. 40.

${ }^{26} \mathrm{~J}$. Ortega y Gasset, Meditaciones del 'Quijote’. Ed. José Luis Villacañas, Madrid: Biblioteca Nueva, 2004, p. 286.

${ }^{27}$ E. M. Wilson, E. M., La vida es sueño, M. Durán (ed.), Calderón y la crítica. Historia y antología, I, Madrid: Gredos, I976, pp. 300-328, aquí: p. 317.

${ }^{28}$ S. Freud, Die Traumdeutung, Köln: Anaconda, 2014, pp. 53, 285; S. Freud, Abriss der Psychoanalyse, Stuttgart: Reclam, 20 Iо, p. 36.

${ }^{29}$ S. Freud, Die Traumdeutung, Köln: Anaconda, 2014, p. 245.
} 
El hecho de que el sueño de Segismundo esté dirigido por la razón y el de César por el inconsciente implica otra diferencia fundamental: mientras Segismundo es, también en el sueño, moralmente responsable de sus acciones, César no lo es. El sueño, dice Freud, no sabe nada de exigencias morales. De este modo, uno no se convierte en

mejor y más virtuoso cuando duerme sino que la conciencia parece callar, más bien, en el sueño, en la medida en que en él no se experimenta compasión y los delitos más graves como crímenes, robos, asesinatos y homicidios pueden ser cometidos con absoluta indiferencia y sin el consecuente arrepentimiento. ${ }^{30}$

Ello explica que César no muestre en la película arrepentimiento por el asesinato de Nuria.

Pero no solo realidad y sueño sino asimismo la determinación frente al libre albedrío es otro de los grandes temas de la obra calderoniana. También en este aspecto se constatan llamativas diferencias entre obra y película derivadas de las ya expuestas anteriormente.

Calderón desarrolla en la obra el artículo cinco de la cuestión novena de la Summa Theologica de Santo Tomás: ${ }^{31}$

Nada prohíbe afirmar que una persona fuere propensa a encolerizarse bajo los influjos de los Astros, o a cualquier otra pasión, ya que por naturaleza la mayoría de los hombres obedece a sus pasiones, a las cuales sólo resisten los sabios. Es por eso que en la mayoría de los casos, es correcto lo que predicen los astrólogos de los actos humanos, después de haber observado el movimiento de los cuerpos celestes. ${ }^{32}$

Por ello, se afirma en La vida es sueño que las estrellas nunca mienten:

\footnotetext{
${ }^{30}$ S. Freud, Die Traumdeutung, Köln: Anaconda, 20 I4, p. 6 I.

${ }^{31}$ Dado que su lectura era obligatoria en los estudios de Teología, es de suponer, por tanto, que Calderón la conocía bien (E. Lorenz, "Calderón und die Astrologie“, Romanistisches Jabrbuch, I 2 (196I), pp. 265-277, aquí: pp. 276-277).

${ }^{32}$ Tomás de Aquino, Summa Theologica, Madrid: Biblioteca de Autores Cristianos, I947, IIae, quest. 9, art. 5.
} 


\section{SEGISMUNDO}

Lo que está determinado

del cielo y en azul tabla

Dios con el dedo escribió, de quien son cifras y estampas

tantos papeles azules

que adornan letras doradas, nunca miente, nunca engaña

porque quien miente y engaña

es quien, para usar mal dellas,

las penetra y las alcanza. ${ }^{33}$

Advierte, sin embargo, Santo Tomás que debe desconfiarse de la Astrología judiciaria porque Dios ha dotado al hombre de razón y, por tanto, siempre queda la posibilidad de que cualquier hombre resista a las pasiones por su libre albedrío: "Hay que tener presente que los mismos astrólogos afirman que el hombre sabio domina a los astros al dominar sus pasiones". ${ }^{34}$ Basilio se equivoca, así, ${ }^{35}$ tratando de vencer al destino con violencia, haciendo, de esta forma, que este se cumpla, ${ }^{36}$ mientras Segismundo ha aprendido la lección de que el destino solo puede vencerse a través de la virtud y de las buenas obras:

\section{SEGISMUNDO}

Sentencia del cielo fue; ${ }^{37}$ por más que quiso estorbarla

\footnotetext{
${ }^{33}$ P. Calderón de la Barca, La vida es sueño, Ed. Fausta Antonucci, Barcelona: Crítica, 2008 , pp. 24I-242.

34Tomás de Aquino, Summa Theologica, Madrid: Biblioteca de Autores Cristianos, I947, Iae, quest. i i 5 , art. 4 .

${ }^{35}$ P. Calderón de la Barca, La vida es sueño, Ed. Fausta Antonucci, Barcelona: Crítica, 2008 , p. I 52.

${ }^{6} \mathrm{P}$. Calderón de la Barca, La vida es sueño, Ed. Fausta Antonucci, Barcelona: Crítica, 2008 , p. I 56.

${ }^{37} \mathrm{~A}$ pesar de que los efectos de las estrellas provengan, según Santo Tomás, de la voluntad de Dios como . ${ }^{\text {er }}$ ster Beweger der Sternen” (E. Lorenz, "Calderón und die Astrologie“, Romanistisches Jabrbuch, I 2 ( I 96 I), pp. 265-277, aquí: pp. 276-277), no cabe la identificación entre esa "sentencia del cielo" que inclina a Segismundo a la tiranía y la voluntad de Dios,
} 


\author{
él [Basilio] no pudo, ¿y podré yo \\ que soy menor en las canas, \\ en el valor y en la ciencia \\ vencerla? 38
}

El final de la obra mostrará que sí. Segismundo vencerá al destino a través de su libre albedrío, eligiendo libremente entre tiranía y virtud. Al optar, finalmente, por la segunda se convertirá en un buen príncipe cristiano. La idea del libre albedrío como argumento para desconfiar de la Astrología judiciaria, pues el hombre siempre está en disposición de cambiar, la encontramos de nuevo en el en Tratado de la verdadera y falsa profecía de Juan de Horozco y Covarrubias de I 588 antes de que reaparezca en el siglo XVII, entre otros, en Calderón:

Y aunque esto sea verdad, que obran en todo lo que les está sujeto y que pueda también obrar el hombre en cuanto al cuerpo y a la compostura dél en

porque este ha dotado a Segismundo de razón y, con ello, de libre albedrío, para resistir esas mismas inclinaciones. La obra, se desmarca, con todo, de la polémica De auxiliis, al no especificarse si Dios ha dotado a Segismundo de auxilio suficiente y depende del propio protagonista que este sea eficaz, tal y como sostiene Suárez (F. Suárez, "En defensa de la Compañía cerca del libre albedrío”, V. Beltrán Heredia (ed.), Domingo Báñez y las controversias sobre la gracia. Textos y documentos, Madrid: Consejo Superior de Investigaciones científicas, I 968, pp. 4r 8-426) o si, tal y como por el contrario afirma Báñez (D. Báñez, "Declaración de P. Domingo Báñez acerca del papel del P. Suárez ”, V. Beltrán Heredia (ed.), Domingo Báñez y las controversias sobre la gracia. Textos y documentos, Madrid: Consejo Superior de Investigaciones científicas, I 968, pp. 426-47 I), el auxilio de Dios recibido por Segismundo es a la vez suficiente y eficaz. Por ello, la obra no puede considerarse ni molinista, tal y como ha afirmado Valbuena Prat, ni tomista, tal y como ha afirmado Lluch sino que solo puede afirmarse, tal y como ha hecho Frutos, su pertenencia a la ortodoxia católica (E. Frutos, La filosofía de Calderón en sus autos sacramentales. Zaragoza: Institución Fernando el Católico, I952, pp. 2 I 4 y ss.) por su defensa del libre albedrío como absolutamente indispensable para la salvación frente al protestantismo, tal y como propone la regla número is a de los Ejercicios espirituales de San Ignacio de Loyola: si en alguna manera y algunas veces se hablare [de la predestinación], así hable que el pueblo menudo no venga en error alguno, como a veces suele diciendo: si tengo que ser salvo o condenado, ya está determinado, y por mi bien hacer o mal no puede ser ya otra cosa; y con esto entorpeciendo se descuidan en las obras que conducen a la salud y provecho espiritual de sus ánimas (I. de Loyola, Ejercicios espirituales, Santander: Sal Terrae, 20 1о, p. 6r).

38P. Calderón de la Barca, La vida es sueño, Ed. Fausta Antonucci, Barcelona: Crítica, 2008 , p. I 55 . 
que se sienten las mudanzas y alteraciones del cielo, no puede tener duda que en las cosas del ánimo a quien Dios crió en libertad no pueden tener fuerza y si alguna es la inclinación se vence con el saber y la discreción $[\ldots]^{39}$

La cuestión entre determinismo y libre albedrío se revela como una de las cuestiones fundamentales del pensamiento occidental. Tanto Sócrates como Platón y Aristóteles consideran que la voluntad está determinada por el conocimiento (solo podemos querer algo malo si no sabemos que es malo y queremos algo bueno porque sabemos que es bueno), una idea para Heimsoeth ${ }^{40}$ asimismo presente en Santo Tomás. La razón está por lo tanto indisolublemente unida a la voluntad y el libre albedrío se define, en consecuencia, como la potestad de actuar por reflexión y elección y es, en este sentido propia, exclusivamente, de los seres humanos. ${ }^{4 \mathrm{I}}$ Desde esta perspectiva, el Segismundo calderoniano dispone en el sueño alegórico de libre albedrío porque, a partir de esa reflexión, y frente a su inclinación, actúa virtuosamente, convirtiéndose, de este modo, en un buen rey. ${ }^{42}$ El sueño de César se desarrolla, sin embargo, de acuerdo a los parámetros freudianos, sin instancia alguna en el subconsciente que someta a los instintos. ${ }^{43}$

El sueño, por tanto, no puede definirse en la pieza teatral y en la película, tal y como propone Xuan, como ámbito de autocreación, ${ }^{44}$ porque César, a diferencia de Segismundo, carece en él de libertad. Aunque el pro-

${ }^{39} \mathrm{~J}$. de Horozco y Covarrubias, Tratado de la verdadera y falsa profecía, Segovia: Juan de la Cuesta, I 588, p. I 32.

${ }^{4} \mathrm{H}$. Heimsoeth, Die sechs großen Themen der abendländischen Metaphysik. Stuttgart: Kohlhammer Verlag, i954, pp. 2 16-2 I 7.

${ }^{4} \mathrm{I}$ E. Tugendhat, "Willensfreiheit und Determinismus", Id., Anthropologie statt Metaphysik, München, Beck, 2007, pp. 57-70. El libre albedrío no debe confundirse, por tanto, con la libertad de acción. Un animal la posee pero no posee libre albedrío. El animal no reflexiona sobre los fines, por lo que no podemos responsabilizarlo moralmente de esa acción.

${ }^{42} \mathrm{P}$. Calderón de la Barca, La vida es sueño, Ed. Fausta Antonucci, Barcelona: Crítica, 2008 , p. 2 I 2.

${ }^{43}$ S. Freud, Die Traumdeutung, Köln: Anaconda, 20 I4, p. 67.

${ }^{44} \mathrm{~J}$. Xuan, "Calderón y el cine: La vida es sueño y Abre los ojos de Alejandro Amenábar", M. Tietz (ed.), Teatro calderoniano sobre el tablado. Calderón y su puesta en escena a través de los siglos, Stuttgart: Franz Steiner Verlag, 2003, pp. pp. 479-49 I, aquí: p. 480. 
tagonista de Amenábar decida libremente comenzar o terminar el sueño no es capaz de determinar, en cambio, libremente su desarrollo. Todo lo que ocurre en el sueño posee una causa, que en principio permanece oculta y que solo al final se nos revela: el recuerdo reprimido del accidente de coche. En el sueño no opera la razón sino el subconsciente, por lo que el protagonista no logra construirlo racionalmente de acuerdo a su voluntad. Con ello, el protagonista se ve condenado a un determinismo radical. Aunque la voluntad de César es vivir un sueño, y con esa intención firma el contrato de Life Extension, el funcionamiento de su propia mente lo transforma inevitablemente en pesadilla. La única opción del protagonista para escapar de ella es abrir los ojos, volver a una realidad, en la que el Yo se ha hecho de nuevo con el control.

Calderón y Amenábar presentan el sueño, por tanto, de forma opuesta. Mientras el sueño es alegórico en Calderón, este es psíquico en Amenábar; mientras en Calderón es la vida terrena la que es sueño, en Amenábar el sueño comienza tras la muerte del protagonista; mientras sueño y realidad están indisolublemente unidos en Calderón, ambas dimensiones se separan, finalmente, en Amenábar; mientras el mensaje de Calderón es que la vida es sueño, el mensaje de Amenábar es que la vida no es sueño; mientras en Calderón el sueño excluye cualquier tipo de determinismo, en Amenábar excluye el libre albedrío.

Todas estas divergencias son resultado, en definitiva, de las diferentes coordenadas espacio-temporales en las que surgen pieza teatral y película. Mientras Calderón escribe su obra en el contexto de la Contrarreforma y asocia el sueño a la cosmovisión barroca, Amenábar traslada la temática calderoniana a la civilización actual y la toma como base para advertir sobre los peligros de una técnica basada en el simulacro, ${ }^{45}$ que con el objetivo de

${ }^{45}$ S. Juan Navarro, S., "La pantalla especular: una lectura metatextual del cine de Alejandro Amenábar“, Letras peninsulares, i6/I (2003), pp. 37 I-384, aquí: p. 37 I. 


\section{diseñar un mundo de ensueño, podría acabar llevando, finalmente, a una existencia pesadillesca. ${ }^{4}$}

\section{BIBLIOGRAFÍA}

Alonso, Dámaso (1976), Poesía española. Ensayo de métodos y límites estilísticos, Madrid: Gredos. Amenábar, Alejandro (1 997), Abre los ojos. Madrid: Sociedad General de Cine.

BáñEz, Domingo (1968), "Declaración de P. Domingo Báñez acerca del papel del P. Suárez", V. Beltrán Heredia (ed.), Domingo Báñez y las controversias sobre la gracia. Textos y documentos, Madrid: Consejo Superior de Investigaciones científicas, pp. 426-47 I.

Calderón de la Barca, Pedro (2008), La vida es sueño, Ed. Fausta Antonucci, Barcelona: Crítica. Freud, Sigmund (2010), Abriss der Psychoanalyse, Stuttgart: Reclam.

Freud, Sigmund (2014), Die Traumdeutung, Köln: Anaconda.

Frutos, Emilio (1952), La filosofía de Calderón en sus autos sacramentales. Zaragoza: Institución Fernando el Católico.

Heimsoet h, Heinz (1 954), Die sechs großen Themen der abendländischen Metaphysik. Stuttgart: Kohlhammer Verlag.

Horozco y Covarrubias, Juan de (I 588), Tratado de la verdadera y falsa profecía, Segovia: Juan de la Cuesta.

IGNACIO DE LOYOla (2OIO), Ejercicios espirituales, Santander: Sal Terrae.

Maule, Rosanna (2007), "Cultural Specificity and Transnational Address in the New Generation of Spanish Film Authors: The Case of Alejandro Amenábar”, C. Sánchez-Conejero (ed.), Spanishness in the Spanish Novel and Cinema of the 2oth - 2 Ist Century, Newcastle: Cambridge Scholars Publishing, pp. I07- I 20.

Juan Navarro, Santiago (2003), "La pantalla especular: una lectura metatextual del cine de Alejandro Amenábar", Letras peninsulares, I6/I, pp. 37 I-384.

Lorenz, Erika (196I), "Calderón und die Astrologie“, Romanistisches Jabrbuch, I 2, pp. 26 5-277.

Ortega y Gasset, José (2004), Meditaciones del 'Quijote’. Ed. José Luis Villacañas, Madrid: Biblioteca Nueva.

Perri, Dennis (2008), “Amenábars Abre los ojos: The post-human subject”, Hispanófila, i 54, pp. 89-98.

Perriam, Chris (2004), "Alejandro Amenábar's Abre los ojos/Open your eyes (1997)”, A. LázaroReboll y A. Willis (eds.), Spanish popular cinema, Manchester: Manchester University Press, pp. 209-22 I.

Pichtoste, Felipe (1 976), "Concepto de la naturaleza deducido de las obras de don Pedro Calderón de la Barca”, M. Durán (ed.), Calderón y la crítica. Historia y antología, I, Madrid: Gredos, pp. 166-248.

Reyes, Alfonso (1957), “Un tema de La vida es sueño”, A. Reyes, Obras completas, VI (pp. I 82-248), México: Fondo de Cultura Económica.

Robertson, Sandra (2000), "Life is virtual dream: Amenábar Reading Calderón", G. CabelloCastellet, J. Martí-Olivella y G. H. Wood (eds.), Cine-Lit 20oo. Essays on Hispanic Film and Fiction, Corvallis: Oregon State University, pp. I I 5- I 25.

${ }^{4}$ Sobre la problematización de la técnica en las sociedades mediales postmodernas que Amenábar desarrolla en Abre los ojos, véase: Carmen Rivero, "Realidad y simulacro: La desmitificación de la técnica en Abre los ojos", José Manuel Losada y Antonella Limpscomb (eds.), Mito y medios audiovisuales, Newcastle: Cambridge Scholars Publishing, [en prensa]. 
SCiaCCA, Michele Federico (1 976), "Verdad y sueño de La vida es sueño de Calderón de la Barca”, M. Durán (ed.), Calderón y la crítica. Historia y antología, I (pp. 541-562). Madrid: Gredos, pp. 54I-562.

Strosetzki, Christoph (2or i), "El Segismundo de Calderón y la segunda naturaleza", Anuario Calderoniano, 4, pp. 333-347.

SuÁrez, Francisco (1968), "En defensa de la Compañía cerca del libre albedrío”, V. Beltrán Heredia (ed.), Domingo Báñez y las controversias sobre la gracia. Textos y documentos, Madrid: Consejo Superior de Investigaciones científicas, pp. 4 1 8-426.

Tomás de Aquino (1947), Summa Theologica, Madrid: Biblioteca de Autores Cristianos.

Tugendhat, Ernst (2007), "Willensfreiheit und Determinismus", Id., Anthropologie statt Metaphysik, München, Beck, pp. 57-70.

Wilson, Edward M. (1 976), La vida es sueño, M. Durán (ed.), Calderón y la crítica. Historia y antología, I, Madrid: Gredos, pp. 300-328.

Xuan, Jing (2003), "Calderón y el cine: La vida es sueño y Abre los ojos de Alejandro Amenábar”, M. Tietz (ed.), Teatro calderoniano sobre el tablado. Calderón y su puesta en escena a través de los siglos, Stuttgart: Franz Steiner Verlag, pp. 479-491.

Ynduráin, Domingo; Rico, Francisco (I 992), "El monólogo de Segismundo”, F. Rico (coord.), Historia y crítica de la literatura española, 3/ I, Barcelona: Crítica, pp. 436-443.

recibido: enero de 2019

aceptado: abril de 2019 